\title{
Tracheal tube obstruction due to hemoptysis associated with pulmonary infarction in a patient with severe COVID-19 pneumonia: A case report.
}

Takaaki Maruhashi ( $\square$ tmaruhasi119@gmail.com )

Department of Emergency and Critical Care Medicine, Kitasato University School of Medicine

\section{Yutaro Kurihara}

Department of Emergency and Critical Care Medicine, Kitasato University School of Medicine

\section{Tatsuhiko Wada}

Department of Rheumatology and Infectious Diseases, Kitasato University School of Medicine

\section{Mayuko Osada}

Department of Emergency and Critical Care Medicine, Kitasato University School of Medicine

\section{Marina Oi}

Department of Emergency and Critical Care Medicine, Kitasato University School of Medicine

\section{Tomonari Masuda}

Department of Emergency and Critical Care Medicine, Kitasato University School of Medicine

Kunihiro Yamaoka

Department of Rheumatology and Infectious Diseases, Kitasato University School of Medicine

Yasushi Asari

Department of Emergency and Critical Care Medicine, Kitasato University School of Medicine

\section{Case Report}

Keywords: COVID-19, pulmonary embolism, case report

Posted Date: September 15th, 2020

DOI: https://doi.org/10.21203/rs.3.rs-75925/v1

License: (1) (1) This work is licensed under a Creative Commons Attribution 4.0 International License. Read Full License

Version of Record: A version of this preprint was published at Cureus on September 15th, 2020. See the published version at https://doi.org/10.7759/cureus.13599. 


\section{Abstract}

Background: The incidence of thrombotic complications is extremely high among severe coronavirus disease 2019(COVID-19) patients in the intensive care unit. Various factors such as a cytokine storm due to an excessive immune response to inflammation, hypoxemia, and disseminated intravascular coagulation are considered predisposing factors for thrombotic complications.

Case presentation: A 55-year-old Japanese man intubated 8 days previously was referred to our hospital because of a severe COVID-19 pneumonia diagnosis after his pharyngeal swab tested positive for severe acute respiratory syndrome coronavirus 2 using reverse transcription-polymerase chain reaction. The patient continued to remain hypoxic $\left(\mathrm{PaO}_{2} / \mathrm{FiO}_{2}\right.$ ratio $\left.<100 \mathrm{mmHg}\right)$ at the referring hospital. On admission, we initiated veno-venous extracorporeal membrane oxygenation (VV-ECMO). Unfractionated heparin and nafamostat mesylate were used as anticoagulants during VV-ECMO. Despite the adequate anticoagulant therapy, he developed pulmonary infarction due to pulmonary embolism followed by hemoptysis. On day 10 following admission, his oxygen saturation dropped from $95 \%$ to $88 \%$, with a marked decrease in his ventilator tidal volume, accompanied by an inability to ventilate the patient. Thereafter, we increased the VV-ECMO flow and exchanged his endotracheal tube. The lumen of the removed tracheal tube was found to be occluded by a large-sized blood coagulum. There was no further episode of tube occlusion. The patient was discharged in a walkable state on day 39 following admission.

Conclusions: Endotracheal tube obstruction secondary to hemoptysis should be suggested in patients with COVID-19 requiring ventilator support, as they are not able to perform frequent endotracheal tube suctions owing to the risk of infection.

\section{Background}

The coronavirus disease 2019(COVID-19) pandemic is yet to show any sign of convergence. COVID-19 causes acute respiratory distress syndrome, and the virus may injure not only the lungs but also various other organs, such as the myocardium, [1] kidney, [2,3] and pancreas [4]. In addition, some recent reports have revealed the risk of embolism in severe COVID-19 cases, which is independent of the state of predisposing thromboembolic risk [5-8]. Herein, we report a rare complication of tracheal tube obstruction in a patient with severe COVID-19 pneumonia and pulmonary embolism.

\section{Case Presentation}

A 55-year-old Japanese man was referred to our hospital because of a severe COVID-19 pneumonia diagnosis after his pharyngeal swab tested positive for severe acute respiratory syndrome coronavirus 2 using reverse transcription-polymerase chain reaction, 8 days following intubation for persistent hypoxia $\left(\mathrm{PaO}_{2} / \mathrm{FiO}_{2}\right.$ ratio $\left.<100 \mathrm{mmHg}\right)$. He had no past history of smoking or any other underlying comorbidity. We initiated the patient on veno-venous extracorporeal membrane oxygenation (VV-ECMO) for providing 
respiratory support. During VV-ECMO, unfractionated heparin (UFH) 10000-15000 units/day and nafamostat mesylate at a fixed infusion rate of $30 \mathrm{mg} / \mathrm{h}$ were provided. Activated partial thromboplastin time (APTT) was maintained in the therapeutic range of 40-60 seconds. Despite the adequate anticoagulant therapy, his D-dimer level was found to be elevated $(87.2 \mu \mathrm{g} / \mathrm{mL})$. Pulmonary embolism involving the right main pulmonary artery and right iliac vein thrombosis around the ECMO cannula insertion site in the right femoral vein were confirmed using contrast-enhanced computed tomography (CECT) (Figure 1a/b). CECT also identified a wedge-shaped infiltrate of poor contrast enhancement along the embolized pulmonary artery, which was consistent with pulmonary infarction. The patient produced a bloody sputum most likely owing to pulmonary infarction. Nursing care including endotracheal tube suctions during VV-ECMO was performed at regular intervals. On day 10 following admission, the patient's oxygen saturation dropped from $95 \%$ to $88 \%$, with a marked reduction in his ventilator tidal volume, accompanied by an inability to ventilate the patient. On attempting endotracheal suctioning, the suction tube did not pass through the endotracheal tube. In response to this situation, we increased the VV-ECMO flow and performed an endotracheal tube exchange. We found that the lumen of the endotracheal tube was occluded by a large-sized blood coagulum. Subsequently, a tracheotomy was performed. There was no further episode of tube occlusion. The patient was taken off VV-ECMO on day 10 and discharged in a walkable state on day 39 of admission.

\section{Discussion And Conclusion}

The incidence of thrombotic complications among severe COVID-19 patients in intensive care units (ICU) is extremely high (31\%) [6]. The predisposing factors for thrombotic complications in COVID-19 patients include various factors such as a cytokine storm due to an excessive immune response to inflammation, hypoxemia, and disseminated intravascular coagulation [9-12].

In our case, the patient was initiated on VV-ECMO and received UFH. The UFH dose was titrated carefully based on APTT monitoring. Our patient developed pulmonary embolism despite these measures. A pulmonary embolism leads to pulmonary infarction, as in this case, and among patients with pulmonary infarction, 13\% develop hemoptysis [13]. The complication of endotracheal tube block by blood coagulum may be explained by the coexistence of hypercoagulability and bleeding tendency against the background of COVID-19 pneumonia. We also considered other possible causes of endotracheal tube obstruction.

The risk of health care workers ( $\mathrm{HCWs)} \mathrm{acquiring} \mathrm{an} \mathrm{infection,} \mathrm{as} \mathrm{they} \mathrm{are} \mathrm{involved} \mathrm{in} \mathrm{the} \mathrm{care} \mathrm{of} \mathrm{patients}$ with COVID-19, is approximately $1.1 \%$. This risk may increase during tracheal tube insertion and endotracheal suctioning, procedures known to generate aerosols [14]. Consequently, it is possible that frequent endotracheal tube suctioning, as is normally recommended in the ICU management of intubated patients with severe COVID-19 pneumonia, may not have been performed in this case. Measures to minimize the risk of an HCW acquiring an infection while performing endotracheal suctioning are warranted as well as increasing their confidence in adhering to the required protocols. This may reduce the incidence of endotracheal tube blockage among patients managed on ventilators. Fortunately, in our 
case, we could exchange the obstructed tracheal tube because of the respiratory assistance provided by VV-ECMO. However, in the absence of VV-ECMO, urgent measures are required for rapid exchange of endotracheal tube.

In patients with severe COVID-19 requiring ventilator support, a high suspicion of endotracheal tube obstruction secondary to various factors should be maintained to avoid adverse outcomes.

\section{Declarations}

Ethics approval and consent to participate: The approval of the ethics committee was waived by the Kitasato University Ethics Committee for this article because this article was a case report and written informed consent was obtained from the patient.

Consent for publication: Written informed consent for this case report was obtained from the patient.

Availability of data and materials: Data sharing is not applicable to this article as no datasets were generated or analyzed during the current study.

Competing interests: The authors declare that they have no conflicts of interest.

Funding: The author(s) received no financial support for the research, authorship, and/or publication of this article.

\section{Authors' contributions:}

Conception and design of study and drafting the manuscript: TM.

Acquisition of data: YK, TW, and MO.

Critical review and revision: All authors

Final approval of the manuscript: All authors

Accountability for all aspects of the work: All authors

Acknowledgements: The authors would like to thank Editage (www.editage.jp) for the English language review.

\section{Abbreviations}

COVID-19: The coronavirus disease 2019

VV-ECMO: Veno-venous extracorporeal membrane oxygenation

UFH: Unfractionated heparin 
APTT: Activated partial thromboplastin time

CECT: Contrast-enhanced computed tomography

ICU: Intensive care unit

HCWs: Health care workers

\section{References}

[1] Chen L, Li X, Chen M, Feng Y, Xiong C (2020) The ACE2 expression in human heart indicates new potential mechanism of heart injury among patients infected with SARS-CoV-2. Cardiovasc Res 116:1097-1100.

[2] Jamie S Hirsch, Jia H Ng, Daniel W Ross, et al (2020) Acute kidney injury in patients hospitalized with COVID-19. Kidney Int S0085-2538(20)30532-9.

[3] Martínez-Rojas MA, Vega-Vega O, Bobadilla NA (2020) Is the kidney a target of SARS-CoV-2? Am J Physiol Renal Physiol https://doi.org/10.1152/ajprenal.00160.2020.

[4] Mukherjee R, Smith A, Sutton R (2020) Covid-19-related pancreatic injury. Br J Surg 107:e190.

[5] Bikdeli B, Madhavan MV, Jimenez D et al (2020) COVID-19 and thrombotic or thromboembolic disease: implications for prevention, antithrombotic therapy, and follow-up. J Am Coll Cardiol 75:2950-2973.

[6] Klok FA, Kruip MJHA, van der Meer NJM et al (2020) Incidence of thrombotic complications in critically ill ICU patients with COVID-19. Thromb Res 191:145-147.

[7] Chen N, Zhou M, Dong X, et al (2020) Epidemiological and clinical characteristics of 99 cases of 2019 novel coronavirus pneumonia in Wuhan, China: a descriptive study. Lancet 395:507-13.

[8] Tang N, Li D, Wang X, et al (2020) Abnormal coagulation parameters are associated with poor prognosis in patients with novel coronavirus pneumonia. J Thromb Haemost 18:844-847.

[9] Chen T, Wu D, Chen H (2020) Clinical characteristics of 113 deceased patients with coronavirus disease 2019: retrospective study. BMJ 368:m1091.

[10] Guan WJ, Ni ZY, Hu Y (2020) Clinical characteristics of coronavirus disease 2019 in China. N. Engl. J. Med 382:1708-1720.

[11] Wang D, Hu B, Hu C (2020) China; Jama: 2020. Clinical characteristics of 138 hospitalized patients with 2019 novel coronavirus-infected pneumonia in Wuhan. JAMA 323:1061-1069.

[12] Zhou F, Yu T, Du R (2020) Clinical course and risk factors for mortality of adult inpatients with COVID19 in Wuhan, China: a retrospective cohort study. Lancet 395:1054-1062. 
[13] Casey K, Iteen A, Nicolini R, Auten J. (2020) COVID-19 pneumonia with hemoptysis: Acute segmental pulmonary emboli associated with novel coronavirus infection. Am J Emerg Med 8:S0735-

6757(20)30239-4.

[14] Xiaoquan Lai, Minghuan Wang, Chuan Qin, et al (2020) Coronavirus disease 2019 (COVID-2019) infection among health care workers and implications for prevention measures in a tertiary hospital in Wuhan, China. JAMA Netw Open 3:e209666.

\section{Figures}
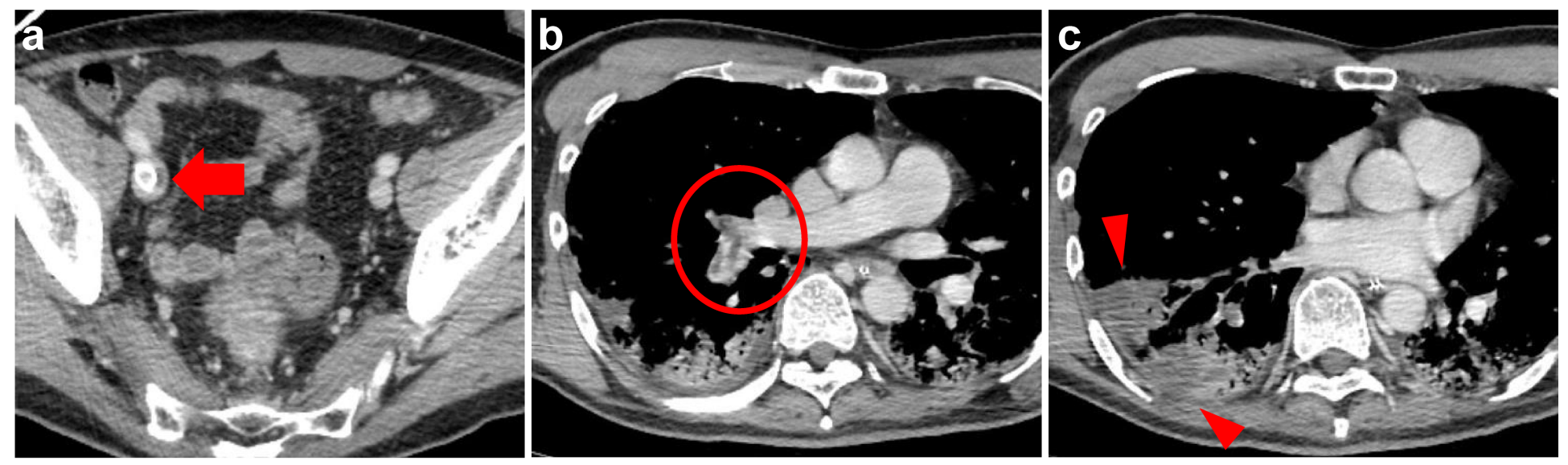

\section{Figure 1}

Chest-pelvis contrast-enhanced computed tomography images (a), (b) Deep vein thrombosis of the right iliac vein around the drainage cannula for extracorporeal membrane oxygenation (arrow), and pulmonary embolism of right main pulmonary artery (circle). (c) Pulmonary infarction, most likely secondary to a pulmonary embolism owing to the presence of poor contrast over a wedge along the embolized pulmonary artery region (arrowhead). 

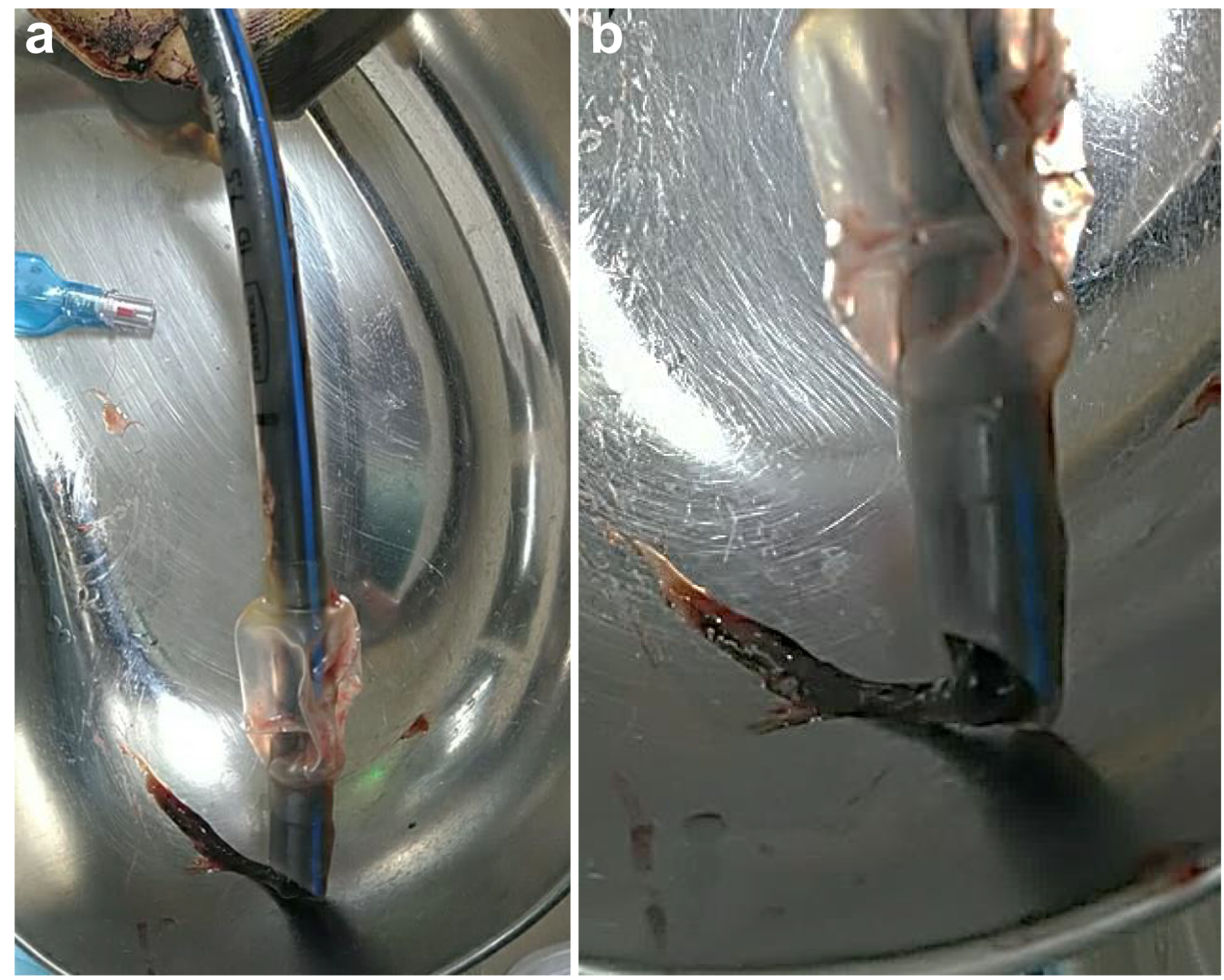

\section{Figure 2}

Removed tracheal tube (a) Total length of tracheal tube indicates the size of the coagulum. (b) Enlarged image of the tracheal tube tip. On day 10, the patient's tidal volume and saturation drops to extremely low levels. A tracheal tube exchange is performed, and ventilation failure is resolved. The tube lumen mostly occluded by blood coagulum. 